\title{
Novel Phases of Planar Fermionic Systems.
}

\author{
Ganpathy Murthy ${ }^{1}$ and R.Shankar ${ }^{2}$ \\ ${ }^{1}$ Departments of Physics Boston University, Boston MA 02215 \\ ${ }^{2}$ Yale University, New Haven, CT 06511
}

(December 7, 2017)

\begin{abstract}
We discuss a family of planar (two-dimensional) systems with the following phase strucure: a Fermi liquid, which goes by a second ordertransition (with non classical exponent even in mean-field) to an intermediate, inhomogeneous state (with nonstandard ordering momentum), which in turn goes by a first order transition to a state with canonical order parameter. We analyze two examples: (i) a superconductor in a parallel magnetic field (which was discussed independently by Bulaevskii)for which the inhomogeneous state is obtained for $1.86 T_{c} \stackrel{\sim}{\sim} B \stackrel{\sim}{<} 1.86 \sqrt{2} T_{c}$ where $T_{c}$ is the critical temperature (in Kelvin) of the superconductor without a field and $B$ is measured in Tesla, and (ii) spinless (or, as is explained, spin polarized) fermions near half-filling where a similar, sizeable window (which grows in size with anisotropy) exists for the intermediate CDW phase at an ordering momentum different from $(\pi, \pi)$. We discuss the experimental conditions for realizing and observing these phases and the Renormalization Group approach to the transitions.
\end{abstract}


murthy@buphy.bu.edu

shankar@cmphys.eng.yale.edu 


\section{INTRODUCTION}

Zero temperature quantum critical phenomena have been a subject of intense theoretical and experimental investigation in the recent years. Of particular interest to us has been the Renormalization Group (RG) description of the transitions. In our pursuit of the RG description of two-dimensional fermionic systems, we came across a family of systems which exhibit similar phase structure and similar phase transitions separating the phases in meanfield theory. Since this phase structure seems generic and experimentally accessible today, we have chosen to make it the focus of this paper, only briefly discussing the RG program that led us to them in the first place.

The systems in question exhibit three phases as a control parameter $I$ is varied. Since the physics depends on the ratio $W / I$, where $W$ is the interaction strength, we can either vary $I$ at fixed $W$ (which is what happens in experiment), or vary $W$ at fixed $I$ in a theoretical discussion. The phases of the system are a weak coupling (large $I$ ) Fermi liquid phase, and a strong coupling phase with conventional order, separated by an intermediate, inhomogeneous phase with nonstandard ordering momentum. The intermediate phase, which is the most interesting, is accessible over a broad window in I (in clean systems) thanks to two-dimensionality. Indeed, in anisotropic systems that are more one-dimensional, the window is broader.

We will illustrate our theme using two concrete examples: a system of electrons with an attractive coupling between up and down spins and a system of spinless fermions with nearest neighbor repulsion. (We hasten to add that we will indicate how these "spinless fermions" are to be realized experimentally by polarizing ordinary electrons in a manner to be specified.)

Consider first the system of free electrons - by which we always mean Landau's quasiparticles - with a circular Fermi surface (FS). They are unstable to the BCS state at arbitrarily small attraction $W$. The instability may be traced back to the time-reversal symmetry of the spectrum and hence of the FS: $E(\vec{K})=E(-\vec{K})$. 
Consider next a system of free spinless fermions, at half-filling, with nearest neighbor hopping on a rectangular lattice. This system is unstable to arbitrarily weak nearest neighbor repulsion $W$ which drives it to a Charge Density Wave (CDW) state at momentum $\vec{Q}=$ $(\pi, \pi)$. The immediate instability is again due to a special symmetry of the FS: due to particle-hole symmetry and nesting, if $\vec{K}$ lies on the Fermi surface, so does $\vec{K}+\vec{Q}$.

Both

instabilities have a nice RG description. It was shown in Reference [1] that in an RG scheme in which modes around the Fermi surface are symmetrically eliminated in thin slices, the Fermi liquid appears as a fixed point and the above mentioned interactions appears as a marginally relevant terms. The existence of a steady flow of the coupling in the simpleminded mode elimination scheme was also seen to result from the special symmetry of the Fermi surface.

We started by asking if such an RG description could be extended to transitions at non-zero coupling. A necessary prelude to such an enterprise was finding systems which exhibited such transitions. It was at that stage that we ran into the systems discussed here.

Consider first the superconductor. To move the transition to a nonzero, but small coupling, (which will allow a perturbative RG analysis ), we must destroy the time-reversal symmetry of its FS by a small amount. To this end, let us apply a magnetic field $B$ parallel to the plane. This parallel field has no effect on the orbital motion but causes a Zeeman splitting of size $2 \mu B \equiv 2 I$, where $\mu$ is the electron's magnetic moment. We expect that at very small fields, the superconductor will be stable, while for very large fields the Fermi liquid will be stable.

Our strategy for showing that there exists an intermediate inhomogeneous phase is as follows. We start at the BCS end and ask when the BCS state yields to the Fermi liquid as the field is raised. Then we go the Fermi liquid end at large $I$ and ask what happens to it as the field is lowered. We find that it first becomes unstable to inhomogeneous pairing before the BCS state beats it in energy. There is clearly a window when neither the BCS nor the Fermi liquid can be the ground state. Here the inhomogeneous state prevails and it 
is to this state, and not the Fermi liquid that the BCS state succumbs as the field is raised.

Here are some details on the implementation of our strategy. Consider the response of the BCS paired state to a small magnetic field $B$. Since the spins form a singlet they ignore the field altogether. In the meantime, as $B$ increases, the Fermi liquid energy decreases because it is getting polarized. Beyond the Chandrasekhar-Clogston [2] limit $I_{F}=\Delta_{0} / \sqrt{2}$ (where $\Delta_{0}$ is the BCS order parameter in the absence of the field), the Fermi liquid has lower energy than the BCS state and one may expect a first order transition (hence subscript $F$ on $\left.I_{F}\right)$ to the polarized Fermi liquid (PFL). But what happens is that by this point a state with inhomogeneous order has beaten the Fermi liquid, and the first order transition is really to this state.

To see all this, so let us go to the weak coupling end (large $I$ ) where it is natural to first find the FS in the presence of the field and then turn on the attraction. The radii of the up and down FS's now differ by $q=2 I / v_{F}$, where $v_{F}$ is the Fermi velocity, hereafter set equal to unity. The PFL is stable to pairing fluctuations, at least for small coupling or large $I$. If we perform the sum over Cooper bubbles and evaluate the pairing susceptibility as $I$ is lowered, we find two interesting features:

- The PFL susceptibility first diverges at $I_{q}=\Delta_{0}$, which is $\sqrt{2}$ times bigger than the $I_{F}$ at which the BCS energy rises above that of the PFL.

- This pairing is at momentum $q=2 I$.

Thus we have a window

$$
\Delta_{0} / \sqrt{2}=I_{F}<I<\Delta_{0}=I_{q}
$$

when the BCS state has a higher energy that the PFL, which itself is unstable to the inhomogeneous state. To repeat, as $I$ is increased, the BCS state will yield, by a first order transition, not to the PFL, but to an inhomogeneous state with wave number $q \simeq 2 I$. The phase diagram is shown in Figure 1a. Actually the inhomogeneous state will prevail even slightly below the lower limit of the above window, since at the first order transition the BCS state has to beat, not the PFL, but the inhomogeneous state 
which has a lower energy. (For completeness we mention that at a still lower field, $I_{0}=\Delta_{0} / 2$ the $q=0$ susceptibility of the Fermi liquid diverges. This is however not very significant since the corresponding instability is preempted by the other transitions. )

Now for the spinless fermions. Here we destroy the perfect nesting of the FS by introducing a chemical potential $I$. (This possibility was raised in Reference [1] along with another option: adding a second neighbor hopping term, which we will consider only briefly.) We establish the window as before. Let us start at strong coupling (small $I$ ), and consider a CDW state at half-filling, with an order parameter $\Delta_{0}$. Now imagine modifying the chemical potential. The system does not respond to a change in the chemical potential which is moving in the gap, exactly the way the superconductor did not respond to the applied field. Once again a mean-field calculation shows that the $(\pi, \pi)$ CDW and the FL have equal energies at $I_{F}=\Delta_{0} / \sqrt{2}$, where $\Delta_{0}$ is the condensate without the chemical potential term. . At the weak coupling end, the FS has shrunk, as shown in Figure 2, and becomes stable against particle-hole pairs condensing at $\vec{Q}=(\pi, \pi)$ or any other momentum. An RPA calculation shows that as $I$ is lowered, the FL becomes unstable to a CDW at a momentum different from $(\pi, \pi)$ at $I_{p} \approx r \Delta_{0}$, where $r>1$ is an anisotropy parameter which measures the ratio of the hopping in the $y$ and $x$ directions. Thus we have a window $\Delta_{0} / \sqrt{2}<I \simeq r \Delta_{0}$ when an inhomogeneous state must prevail. This state is inhomogeneous in the sense that it is characterized by a momentum different from the canonical value, which in this case happens to be $(\pi, \pi)$. The phase diagram is shown in Figure 1b. (The significance of the points $I_{q}$, and $I_{0}$ which do not correspond to transitions, will be explained in section III.)

In both the BCS and CDW cases the mean-field second order transition from the FL to the inhomogeneous state was found to have non-classical exponents. For example $\beta=2$ for the superconductor. This nonclassical answer in a mean-field calculation may be ascribed to the fact that the mode that becomes unstable has a momentum which is a singular point of the susceptibility. This singularity and the existence of a fairly large window for the inhomogeneous phase are both a result of the two-dimensionality of the system.

The plan of the paper is as follows: In the next section we will discuss the superconductor 
and relate it to earlier work. In trying to track down existing literature in the case of the superconductor, we were invariably directed to the works of Larkin and Ovchinnikov, and Fulde and Ferrel [3], and we shall briefly discuss these. However, upon digging further, we found some work of Bulaevskii [5] to be far more relevant, as will be discussed. In section III we will perform a similar analysis of the spinless fermion system and describe how it may be experimentally realized. Section IV is devoted to the status report of the RG program. We end with conclusions and avenues for future research in section $\mathrm{V}$.

\section{THE PLANAR SUPERCONDUCTOR}

We will begin with noninteracting electrons lying within an annulus of thickness $2 \Lambda$ centered around the Fermi circle of radius $K_{F}$. We will construct a mean-field hamiltonian that pairs the up and down electrons whose momenta lie within this band.

The energy of an up electron is

$$
E_{+}(\vec{K})=I+\left(\frac{K^{2}-K_{F}^{2}}{2 m}\right)=I+v_{F} k=I+k
$$

where the Fermi velocity $v_{F}$ has been set equal to unity, and

$$
k=K-K_{F} .
$$

Terms higher order in $k$ are dropped.

The energy of its partner, a down spin electron of momentum $-\vec{K}+\vec{q}$, is

$$
E_{-}(-\vec{K}+\vec{q})=-I+k-q \cos \theta
$$

where $\theta$ is the angle between $\vec{K}$ and $\vec{q}$.

(It is understood that $-\vec{K}+\vec{q}$ is also constrained to lie in the same annulus. This means that certain electrons will not have partners for pairing in a state of momentum $q$. They must be handled correctly.)

A useful combination is 


$$
\frac{E_{+}(\vec{K})-E_{-}(-\vec{K}+\vec{q})}{2}=I+(q / 2) \cos \theta \equiv z(\theta) .
$$

Let us begin with the mean-field hamiltonian

$$
\begin{aligned}
H= & \frac{\Delta \Delta^{*}}{W} \\
& +\int_{-\Lambda}^{\Lambda} d k \int_{0}^{2 \pi} d \theta\left[c_{+}^{\dagger}(\vec{K}) c_{+}(\vec{K})(I+k)+c_{-}^{\dagger}(\vec{K}) c_{-}(\vec{K})(-I+k)\right. \\
& \left.-\Theta\left(\Lambda-|| \vec{K}-\vec{q}\left|-K_{F}\right|\right)\left[\Delta c_{+}^{\dagger}(-\vec{K}+\vec{q}) c_{-}^{\dagger}(\vec{K})+\Delta^{*} c_{-}(\vec{K}) c_{+}(-\vec{K}+\vec{q})\right]\right]
\end{aligned}
$$

where the condensate was taken to have the form $\Delta(x)=\Delta \exp [i \vec{q} \cdot \vec{r}]$, the step function $\Theta\left(\Lambda-|| \vec{K}-\vec{q}\left|-K_{F}\right|\right)$ ensures that the other partner in the pair also lies in the annulus, and the subscripts refer to the spin. The reader should not worry about factors of $2 \pi$ since all key results will be given in term of observables.

By going to Bogolubov operators $d_{ \pm}$and performing the usual transformation that kill the "bad" terms, we obtain

$$
\begin{aligned}
H= & \frac{\Delta \Delta^{*}}{W} \\
+ & \int_{-\Lambda}^{\Lambda} d k \int_{0}^{2 \pi} d \theta \Theta\left(\Lambda-|| \vec{K}-\vec{q}\left|-K_{F}\right|\right) \\
& {\left[k-I-q \cos \theta+d_{+}^{\dagger} d_{+}\left[I+(q / 2) \cos \theta+\sqrt{\Delta^{2}+(k-(q / 2) \cos \theta)^{2}}\right]\right] } \\
& +\left(d_{-}^{\dagger} d_{-}-1\right)\left[-I-(q / 2) \cos \theta+\sqrt{\Delta^{2}+(k-(q / 2) \cos \theta)^{2}}\right]+\frac{\pi q^{2}}{2}-4 \Lambda q .
\end{aligned}
$$

The last two terms come from the unpaired electrons. The ground state is found by filling all negative energy states. (If we set $\Delta=0$ above, the ground state energy should reduce to $-2 \pi\left(\Lambda^{2}+I^{2}\right)$, the energy of the PFL. ) If we subtract off the energy of the PFL, we find the relative energy

$$
\begin{aligned}
E(\Delta)-E(0)= & 2 \pi I^{2}+\frac{\Delta^{2}}{W}+\pi\left(\frac{q^{2}}{4}-\Delta^{2}\right)-2 \pi \Delta^{2} \ln \frac{2 \Lambda}{\Delta} \\
& +\int_{0}^{2 \pi} d \theta\left[\theta(z-\Delta)\left[-z \sqrt{z^{2}-\Delta^{2}}+\Delta^{2} \ln \frac{z+\sqrt{z^{2}-\Delta^{2}}}{\Delta}\right]+z \rightarrow-z\right] \\
z(\theta)= & I+\frac{q}{2} \cos \theta
\end{aligned}
$$

where $\Delta$ and $\Delta^{2}$ stand for $|\Delta|$ and $|\Delta|^{2}$. 
Most of our results are based on an analysis of this equation.

The first term comes from subtracting off the polarization energy of the PFL. As for the $\theta$ integral let us note that if $\Delta>I+q / 2$, it does not contribute. It is clear that nonzero $q$ is a liability in this region and the minimum energy is readily found to occur at $\Delta_{0}$ given by

$$
\frac{1}{W}=2 \pi \ln \frac{2 \Lambda}{\Delta_{0}}
$$

exactly as if $B$ were never turned on. This is just the manifestation of the fact that the BCS system ignores the field. The condensation energy (relative to the PFL energy) is found to be

$$
E(\Delta)-E(0)=\pi\left(2 I^{2}-\Delta_{0}^{2}\right)
$$

which leads to the result that $I_{F}=\Delta_{0} / \sqrt{2}$ is the field at which the BCS system yields to the Fermi liquid. (Since $\Delta_{0}=\sqrt{2} I_{F}>I_{F}$ the answer is consistent with the assumptions made in deriving it.)

Now for the weak coupling (or large $I$ ) Fermi liquid end. Either by summing all the Cooper bubbles, or by taking the $\Delta^{2}$ derivative of the energy at $\Delta=0$, we find the formulas for $\chi^{-1}$. (In taking this derivative it is important to note that if $I>(q / 2), z(\theta)$ is always positive and only the first term in the $\theta$ integral contributes, whereas if $(q / 2)>I$, the second contributes for $\Delta=0$.) The result is

$$
\begin{array}{rlrl}
\chi^{-1}((q / 2)) & =\frac{1}{W}-\int_{0}^{2 \pi} d \theta \ln \frac{\Lambda}{|z(\theta)|} & \\
& =\frac{1}{W}-2 \pi \ln \frac{2 \Lambda}{I+\sqrt{I^{2}-(q / 2)^{2}}} & (q / 2)<I \\
& =\frac{1}{W}-2 \pi \ln \frac{2 \Lambda}{(q / 2)} \quad(q / 2)>I .
\end{array}
$$

Note that at each value of I (the applied field), the softest mode is the one with $(q / 2)=I$ and that this is a singular point of $\chi$. This mode becomes unstable when $I=I_{q}$, where

$$
\frac{1}{W}=2 \pi \ln \frac{2 \Lambda}{I_{q}}
$$


If we compare this equation to the gap equation in the absence of field

$$
\frac{1}{W}=2 \pi \ln \frac{2 \Lambda}{\Delta_{0}}
$$

we see that

$$
I_{q}=\Delta_{0}
$$

Thus we have a window

$$
\frac{\Delta_{0}}{\sqrt{2}} \stackrel{\sim}{<} I \stackrel{\sim}{<} \Delta_{0}
$$

wherein the neither the Fermi liquid, nor the homogeneous superconductor is the ground state. (Actually the lower limit should be lower since the BCS state has to beat not the PFL but the inhomogeneous state. However the condensation energy of the inhomogeneous state is quite small and so is the change in the lower limit.)

If we put in the numbers we find that if $B$ is in Tesla and $T_{c}$ in Kelvin, the new phase should be stable in the interval $1.86 T_{c} \stackrel{\sim}{<} B \stackrel{\sim}{<} 1.86 \sqrt{2} T_{c}$. We have used here the free electron $g$ factor. In practice $g$ could be much higher, and the values of $B$ correspondingly lower.

Figure 3 shows a plot of the energy in Eqn.(可) versus $\Delta$. The parameters $\Lambda=100, I=$ $.834, W=.031$ are chosen so as to bring the BCS energy (measured relative to the PFL) to zero, i.e., we have chosen $I=\Delta_{0} / \sqrt{2}$. Plotted on the same graph is the energy of the inhomogeneous state at $(q / 2)=.923$. Note that this $(q / 2)$ is not equal to $I$. In other words, although $(q / 2)=I$ becomes unstable first, once the order sets in, a larger $(q / 2)$ does better and the graph shows the energy for the best $(q / 2)$. If we lower $I$ a bit, the BCS state will dip down very quickly to below the inhomogeneous state. Thus we may take $I=\Delta_{0} / \sqrt{2}$ to be essentially the field for the first order transition to the inhomogeneous state. If $I$ is raised further, the BCS graph will move upwards and so will the inhomogeneous state's energy at the minimum. As the minimum moves towards the origin, the optimal $(q / 2)$ will move towards the current value of $I$. Finally for $I>\Delta_{0}$ the PFL will become stable to all pairing fluctuations. The transition to the PFL is clearly second order in mean-field theory. 
From the energy formula it is easy to compute the specific heat by finding the density of states, and the magnetization by taking the $I$ derivative. (Since $(q / 2)$ and $\Delta$ are slaved to $I$ one may ask how they are to be handled during the derivation. In general there will be implicit and explicit changes in $E$ to to a change of $I$, however at a point where $\partial E / \partial q$ and $\partial E / \partial \Delta$ vanish (as they do for us) only the explicit derivative matters.) The result is, in terms of the corresponding quantities for the PFL,

$$
\begin{aligned}
\frac{M}{M_{P F L}} & =\frac{1}{2 \pi I} \int_{0}^{2 \pi} d \theta \sqrt{z^{2}-\Delta^{2}}[\theta(z-\Delta)-\theta(-z-\Delta)] \\
\frac{C}{C_{P F L}} & =\frac{1}{2 \pi} \int_{0}^{2 \pi} d \theta \frac{[z \theta(z-\Delta)-z \theta(-z-\Delta)]}{\sqrt{z^{2}-\Delta^{2}}}
\end{aligned}
$$

where $\Delta$ and $(q / 2)$ are at their optimal values. Putting in the numbers we find that these ratios drop from the value of unity at the PFL end, down to .92 and .79 respectively by the time we reach the situation shown in Figure 3 (which is more or less the end of the inhomogeneous phase).

Let us next compute the exponent $\beta$ which characterizes the onset of $\Delta$. If we could write the energy near the transition as

$$
E=-t \Delta^{2}+u \Delta^{4}+\ldots
$$

where $t=\Delta_{0}-I$, we would find as usual that $\Delta \simeq t^{1 / 2}$. If however we analyze our energy function we find that

$$
E=-t \Delta^{2}+u \Delta^{5 / 2}+\ldots
$$

leading to $\beta=2$. In other words, the energy function does not have an analytic expansion in $\Delta$ near the transition, which in turn can be traced to the fact that the optimal $(q / 2)$ at the transition is a singular point of $\chi^{-1}$. Thus even though we are doing mean-field theory, a nonclassical exponent emerges.

We could go on and compute the electromagnetic response functions but do not, for the following reasons. Our analysis has shown that in the window 


$$
\frac{\Delta_{0}}{\sqrt{2}} \stackrel{\sim}{\sim} \stackrel{\sim}{<} \Delta_{0}
$$

an inhomogeneous state characterized by momentum of magnitude $q$ (which is of the order I) has lower energy than either the PFL or the BCS state. However our analysis does not choose a particular direction for $q$. Thus, as in problems involving, say crystallization, 66 all modes in this circle are degenerate. In this case the low energy physics of the condensate is very much like that of a Fermi system, i.e., centered around a circle and not the origin. Recently Hohenberg and Swift [7] have shown how the shell elimination devised in Ref. [1] for fermions can be applied to this problem. For the present simply note that, instead of the plane wave we considered so far, a standing wave of the same wavelength might have a lower energy. The standard trick for deciding what exactly happens is to expand the energy functional in a power series in $\Delta(\vec{q})$, going out to fourth order. The fourth order vertex (which is the particle-particle bubble with two more insertions it it) will determine which combination of modes is best the near transition. Unfortunately we cannot do this here since an analytical expansion in $\Delta$ does not exist. While we may expect that $\beta=2$ may be insensitive to the actual form of the order parameter (plane wave versus cosine), features like specific heat will be very sensitive to the true ground state. For example if $\Delta \simeq \cos q r$, bands will be formed and the density of states can go up above the PFL value. The only thing that seems certain is that the specific heat will not be activated. Likewise the electromagnetic response and Meissner effect (for a small perpendicular field) will depend on the knowledge of the true ground state. This is a problem we have not solved. All we can say is that if the answer is a cosine, there will be an oscillation in spin density at the same wavelength.

We now consider the relation of our work to that of Larkin -Ovchinnikov and Fulde-Ferrel [3] [四. These authors considered a three dimensional superconductor with ferromagnetic impurities. The spins of these impurities coupled to the electrons via the exchange interaction so that they could be represented on the average by some external field that coupled only to spin. These authors too predict an inhomogeneous phase. We now list the differences 
between our work and theirs.

- These authors consider a system coupled to magnetic impurities while we need a clean system.

- They evade the orbital complication of the magnetic field (even though they work in three dimensions) because the field really represents the exchange interaction with impurities. We evade it by considering a planar system with an external parallel field.

- Most importantly, they expect the inhomogeneous phase over a very narrow window $.707 \Delta_{0} \leq I \leq .755 \Delta_{0}$ in three dimensions whereas we expect it over a broad window $.707 \Delta_{0} \leq I \leq \Delta_{0}$ in two dimensions, with $I$ as the controllable external field. The window will be even wider in anisotropic systems (e.g. elliptical FS) because they are more one dimensional and will have roughly parallel segments of the FS over longer intervals.

- Due to the fact that the optimal $q$ is a singular value of $\chi^{-1}$ we find nonclassical exponents $(\beta=2)$ in the mean-field level, while they find classical exponents due to the fact that the optimal $q$, for small $\Delta$ is $q=1.2 I$, which is a nonsingular point of the three dimensional susceptibility. This allowed Larkin and Ovchinnikov to study mode coupling and to decide that the cosine order parameter is better than the plane wave. Till we can do a similar thing here it may be a reasonable to assume that the same thing happens in $d=2$ also. In this case we can expect as they do, that the spin density will will oscillate with period $q$ and that the specific heat will be greater than for the PFL.

Consider next the work of Bulaevskii [5]. He looks at layers in a magnetic field and works out critical fields for any tilt and at finite temperature. His formula for zero tilt at zero temperature gives the window we quoted. The exponent $\beta=2$ is reflected in the $T$ dependence of his results. Like us, he does not consider spin orbit coupling and for this reason could not explain some of the experiments [8] done at that time, as pointed out by 
Klemm, Luther and Beasley [9], who did include this effect. It appears that the time is ripe to see this inhomogeneous phase. The conditions are that the material be as two-dimensional as possible, clean, have low $Z$ (to minimize spin-orbit effects) and have a low $T_{c}$ so that the required fields are are not too large. It will be interesting, not only in its own right, but also as a part of the family of systems discussed here. Of course at finite $T$, there will be no ordered phase and a Kosterlitz-Thouless phase with algebraic order will take its place [10]. The phase structure will still be visible at small $T$ and the $T=0$ critical point will control the finite $T$ physics in the quantum critical region as in other problems [11] [12].

\section{THE SPINLESS FERMION SYSTEM WITH CDW ORDER}

We begin by reassuring our readers that what we mean by a spinless fermion can be experimentally realized in at least one way. In the idealized calculation we consider a system of spinless fermions that fills up a nested Fermi sea and ask what happens as we tamper with the nesting by changing the chemical potential. Assuming that all the action is centered around the immediate vicinity of the Fermi surface, i.e., that the interaction and the change in chemical potential $I$, are small compared to the bandwidth, we can duplicate the same physics near the Fermi surface as follows. We take regular electrons and fill them up to, say, $45 \%$ (instead of half) of the Brillouin zone. Now we apply a magnetic field in the plane. This does nothing to the orbital motion but splits the spin up and down Fermi surfaces. For some choice of field, the bigger of the surfaces will begin to nest, while the other would have moved away in the opposite direction and hopefully will not do anything interesting. The polarized electrons with a nested or nearly nested Fermi surface will be the spinless fermions of the theoretical calculation. Of course a CDW in this case will also imply a spin density wave or SDW.

As in Reference [1] we will consider free fermions with the dispersion relation which comes from nearest neighbor hopping:

$$
E(x, y)=-\cos x-r \cos y
$$


where $x$ and $y$ stand for $K_{x}$ and $K_{y}$. We choose anisotropic hopping since the generic physics we are talking about can be obscured by the van Hove singularities that lie on the Fermi surface when $r=1$. We will work with $r>1$. This energy relation satisfies the condition

$$
E(x+\pi, y+\pi)=-E(x, y)
$$

and the vector $Q=(\pi, \pi)$ connects points on the two branches of the Fermi surface, labeled by $\alpha= \pm 1$ as shown in Figure 2. At half-filling the Fermi surface is defined by $E=0$ or

$$
y= \pm \cos ^{-1}\left[-\frac{\cos x}{r}\right]=\alpha \cos ^{-1}\left[-\frac{\cos x}{r}\right]
$$

We will shortly use a variable $\varepsilon$ that measures the energy from the FS of the free particles. In our problem where the FS is at zero energy, $\varepsilon=E$.

Imagine turning on a chemical potential so that

$$
E(x, y)=-\cos x-r \cos y+I
$$

The Fermi surface of the free fermions will now shrink to a smaller size and no longer nest. Of relevance is the combination

$$
\begin{aligned}
\frac{E(x+\pi+q, y+\pi+p)-E(x, y)}{2} & \equiv z(x, y, q, p) \\
& =I-(q / 2) \sin x-r(p / 2) \sin y \\
& =I-(q / 2) \sin x-(p / 2) \alpha \sqrt{r^{2}-\cos ^{2} x} \\
& \equiv z_{\alpha} .
\end{aligned}
$$

In the above formula, $\alpha= \pm 1$ is the branch index, and, as everywhere else in the paper, terms of quadratic order or higher in the small quantities $I, p, q$ will be ignored.

Once again we limit ourselves to a band of energy $\Lambda$ on either side of the free electron FS. To this end we change variables from $(x, y)$ to $(x, \varepsilon)$, where $\varepsilon$ measures the energy from the FS. (See Reference [1] for details. ) As a result 


$$
\int d x d y \rightarrow \int d x d \varepsilon J(r, x)
$$

where the Jacobian is

$$
J(r, x)=\frac{1}{\sqrt{r^{2}-\cos ^{2} x}}
$$

on the free electron FS. We will ignore its deviation off the FS. It is useful to define

$$
\bar{J}=\frac{1}{2 \pi} \int_{0}^{2 \pi} J(x) d x
$$

The mean-field hamiltonian is

$$
\begin{aligned}
H= & \left.\frac{\Delta^{2}}{W}+\int_{-\Lambda}^{\Lambda} d \varepsilon \int_{0}^{2 \pi} d x J(r, x)\left[c_{+}^{\dagger}(\varepsilon, x) c_{+}(\varepsilon, x)+c_{-}^{\dagger}(\varepsilon, x)\right) c_{-}(\varepsilon, x)\right] \\
& +\int_{-\Lambda}^{\Lambda} d \varepsilon \int_{0}^{2 \pi} d x J(r, x) \Theta(|\varepsilon(x+\pi+q, y+\pi+p)|<\Lambda) \\
& {\left[\Delta\left(c_{+}^{\dagger}(\vec{K}) c_{-}(\vec{K}+\vec{Q}+\vec{i} q+\vec{j} p)+\Delta^{*} c_{-}^{\dagger}(\vec{K}+\vec{Q}+\vec{i} q+\vec{j} p) c_{+}(\vec{K})\right]\right.}
\end{aligned}
$$

where now the subscripts \pm on the operators refer to the branches of the FS.

To exhibit the intermediate phase, let us assemble the two ingredients: (i) The energy difference between the Fermi liquid and the $(\pi, \pi)$ CDW state and (ii) The inverse Fermi liquid susceptibility (in RPA) to CDW formation at momentum $(x+\pi+q, y+\pi+p)$. The space between the zeros of these gives a window in $I$ for the inhomogeneous state.

A standard mean-field calculation, as in the superconducting problem, shows that for $\Delta>I$, the energy difference between the $(\pi, \pi)$ CDW state and Fermi liquid is

$$
E(\Delta)-E(0)=\frac{\Delta^{2}}{W}+\int_{0}^{2 \pi} d x J(r, x)\left[I^{2}-\frac{\Delta^{2}}{2}-\Delta^{2} \ln \frac{2 \Lambda}{\Delta}\right]
$$

This energy difference has a minimum at

$$
\Delta_{0}=2 \Lambda \exp \left[-\frac{1}{2 \pi \bar{J} W}\right]
$$

Feeding this back into the energy formula we find that the $I$ at which the $(\pi, \pi)$ CDW state loses out to the Fermi liquid is

$$
I_{F}=\frac{\Delta_{0}}{\sqrt{2}}
$$


(Note that once again the answer is consistent with the assumption $\Delta>I$ that went into its derivation.)

By summing the usual particle hole bubbles, the inverse susceptibility of the Fermi liquid is found to be

$$
\chi^{-1}(q, p)=\frac{1}{W}-\frac{1}{2} \sum_{\alpha} \int_{0}^{2 \pi} d x J(r, x) \ln \frac{\Lambda}{\left|z_{\alpha}\right|}
$$

where $z_{\alpha}$ is the value of $z$ on branch $\alpha$, as defined in Eqn.(29). This gives

$$
\chi^{-1}(q, p)=\frac{1}{W}-\int_{0}^{2 \pi} d x J(r, x) \ln \frac{\Lambda}{\sqrt{(I-(q / 2) \sin x)^{2}-(p / 2)^{2}\left(r^{2}-\cos ^{2} x\right)}}
$$

First note that the uniform CDW (i.e., $(\pi, \pi)$ ) mode becomes singular when $I=I_{0}$ where

$$
\frac{1}{W}=2 \pi \bar{J} \ln \frac{\Lambda}{I_{0}}
$$

i.e., at

$$
I_{0}=\Lambda \exp \left[-\frac{1}{2 \pi \bar{J} W}\right]=\frac{\Delta_{0}}{2} .
$$

Next we consider just nonzero $q$. The instability occurs when

$$
\frac{1}{W}=\int_{0}^{2 \pi} J(r, x) d x \ln \frac{\Lambda}{|I-(q / 2) \sin x|} .
$$

Let us consider this equation at large $r$ where the analysis is easier. Now $J \simeq 1 / r$ and

$$
\begin{aligned}
\frac{1}{W} & =\frac{2 \pi}{r} \ln \frac{2 \Lambda}{I+\sqrt{I^{2}-(q / 2)^{2}}} \quad q / 2<I \\
& =\frac{2 \pi}{r} \ln \frac{2 \Lambda}{(q / 2)} \quad q / 2>I .
\end{aligned}
$$

The optimal value is $q / 2=I$, and we find upon inverting the above that the $I$ value at the onset of the instability is

$$
I_{q}=2 \Lambda \exp \left[-\frac{r}{2 \pi W}\right]=2 \Lambda \exp \left[-\frac{1}{2 \pi \bar{J} W}\right]=\Delta_{0}
$$

At $r$ not too large, but also not too close to unity (so that the singularity of the Jacobian does not dominate the integral), similar results hold. 
So far we have found the same window as in the case of the superconductor, namely

$$
\frac{\Delta_{0}}{\sqrt{2}}<I<\Delta_{0}
$$

But the window gets wider if we look at nonzero $p$. (This is also clear from Figure 2.) Now we have

$$
\begin{aligned}
\frac{1}{W} & =\int_{0}^{2 \pi} J(r, x) d x \ln \frac{\Lambda}{\sqrt{I^{2}-(p / 2)^{2}\left(r^{2}-\cos ^{2} x\right)}} \\
& =\int_{0}^{2 \pi} J(r, x) d x \ln \frac{\Lambda}{\sqrt{\left.I^{2}-(r p / 2)^{2}+\left(p^{2} / 8\right)+\left(p^{2} / 8\right) \cos 2 x\right)}}
\end{aligned}
$$

For large $r$, this integral is seen to be a maximum when $p=2 I / r$ where its value is $(2 \pi / r) \ln \frac{2 \Lambda r}{I_{p}}$ so that

$$
I_{p}=r \Delta_{0} .
$$

The following table shows the deviation from this result for smaller $r$. In all these cases the optimal $p$ was found to be $2 I / r$. Note that the large $r$ results are not bad for $r$ as low as 1.1.

\begin{tabular}{|c|c|c|c|c|}
\hline$r$ & 1.1 & 1.5 & 2.5 & 3.5 \\
$\frac{I_{p}}{r \Delta_{0}}$ & .83 & .93 & .98 & .99 \\
\hline
\end{tabular}

The final phase diagram is as shown in Figure 1b. The main point is that as the anisotropy grows, so does the window, since the system is becoming increasingly one dimensional and the opposite faces of the Fermi surface are becoming globally parallel. (In the limit of parallel faces there exists a perfect nesting vector for any $I$.)

We will not consider the general case of a nonzero $q$ and $p$ since we have already seen a substantial window for observing the inhomogeneous phase. We also do not try to compute its energy as a function of $\Delta$ (as we succeeded in doing for the superconductor) because of the following problem. Recall that in the superconductor problem we coupled $K$ to $-K+q$. That in turn must be coupled to $-(-K+q)+q=K$, which means only two modes are 
to be coupled. In the present case we coupled $K$ to $K+(\pi, \pi)+q$, which in turn must be coupled to $K+2 q$ and so on. We did perform a calculation keeping just the first coupling to exhibit a variational wave function which could beat the Fermi liquid and the $(\pi, \pi)$ state inside the window.

Due to the fact that the optimal momentum $p$ at the transition is a singular point of $\chi$, we can once again expect nonclassical exponents. We guess that $\beta$ will be larger than the classical value, probably equal to 2 once again.

\section{A. Experimental realization}

Let us now turn to the experimental realization of this system. We start with a system of real electrons not too close to half-filling, say at $45 \%$ filling. At zero temperature, this will probably be a BCS superconductor. Next we apply a parallel magnetic field. This is the problem we just studied. The BCS state will eventually pass via the inhomogeneous superconducting phase to the polarized Fermi liquid as the field rips apart the Fermi surfaces of the up and own spins. (Here we must choose a material with a large $g$ factor for the electron.) Focus on the two Fermi surfaces. One shrinks further away from half-filling and hopefully does nothing interesting. The FS of the growing species will now come close to nesting as the field is raised. This species of polarized electrons constitutes the spinless fermions of the calculation, while the applied field, which adds a constant (Zeeman) energy per particle, will play the role of the changing chemical potential. Note however that as the magnetic field grows and pushes the FS closer to nesting, the $I$ in the spinless version decreases. At one point the system should enter the inhomogeneous CDW phase, which here means the spin density also will oscillate with a momentum slightly different from $(\pi, \pi)$. Eventually the system will jump by a first order transition to the $(\pi, \pi)$ state. In other words, when the chemical potential (i.e.e, applied magnetic field)

falls within a window, the system density locks at half-filling, taking particles from the reservoir, which in this case is the other species of spins. We should see a sudden increase 
in polarization which should then remain fixed as the field is increased, since the up and down densities remain locked. (Unlike in the case of an ideal reservoir which can donate any number of particles at one energy, there will be a cost of energy when a macroscopic number of fermions have to be converted from the shrinking FS to the growing one. This will decrease the region of stability of the commensurate $(\pi, \pi)$ CDW phase.) If the field is raised further, we are effectively changing the sign of $I$ and so the same picture will appear in reverse: a first order transition to the inhomogeneous state followed by a second order transition to the Fermi liquid.

By design, the grand canonical picture applies to the above experiment, where the system of polarized electrons indeed is in contact with a reservoir (of opposite spin electrons). The free parameter is the chemical potential or the applied magnetic field, and the particle density is chosen by the system to minimize its energy.

Now the ideas discussed above also apply to other problems where the number density is the independent variable, say when a system of regular electrons at half-filling, (as in a Hubbard model), is doped. Whereas if we took a commensurate system and changed its chemical potential, it will initially ignore it and hang on its special density, here we want to forcibly change the number density and see what happens. We illustrate what is to be done in such a case by re-expressing the above spinless fermion analysis in terms of number density. In other words we ask- Suppose we took a half-filled system of spinless fermions and doped it, what will it do? Figure 4, which is a schematic, helps us analyze this. Along the $x$-axis we vary $I$, the chemical potential, and cover the three phases. (The CDW phase refers to the $(\pi, \pi)$ state). We find (in our calculation) that it has half-filling for all $I$ up to the first order transition. So we plot the corresponding $n$ as a dark horizontal line ( at $1 / 2$ ) going up to the transition. A slight increase in $I$ causes a first order transition to the inhomogeneous state. It has a lower density $n_{q}$. Further increase in $I$ causes a decline in $n$ till we reach the second order transition to the Fermi liquid. There is no jump in $n$. Hereafter $n$ and $I$ are related as in a free Fermi liquid: $n=\frac{1}{2}-I$. (The coefficient of $I$, the density of states, is set to unity). Now we can answer the question we posed. To find 
out what happens at any given density, choose a value for $n$, move horizontally to the dark line and come right down to the phase diagram on the $I$ axis. Thus when $n=1 / 2$ the system is in the $(\pi, \pi)$ state. As $n$ is lowered, ever so slightly, the system is stuck at the first order transition and there will be coexistence of the $(\pi, \pi)$ CDW and inhomogeneous states, the ratio being determined by $n$. When $n$ is lowered down to $n_{q}$, the system will become all inhomogeneous. Thereafter we move smoothly along the dark line to the Fermi liquid transition and Fermi liquid phase. Thus, although a slight change in chemical potential does nothing to the $(\pi, \pi)$ state, the slightest doping causes phase coexistence.

The above discussion has focused on breaking the nesting symmetry by changing the chemical potential. This is the choice with the closest analogy to the superconductor. We have also studied a case where nesting is destroyed by adding a second neighbor term which causes additional wiggles on the FS without changing its volume. Here we found that the window for the inhomogeneous state shrinks. In the large $r$ limit, it goes to zero: the second order transition at the origin in $\Delta$ space occurs when the first order transition does. It is still interesting that the first order transition far from the origin will be accompanied by huge fluctuations (at an incommensurate momentum) close to the origin. The reason the uniform state is more stable in the presence of this kind of modification of the FS is clear: whereas changing $I$ ruins nesting at $(\pi, \pi)$ uniformly over the FS, the additional term due to second neighbor hopping vanishes at some points on the old FS. Indeed any modification to the dispersion relation will necessarily be periodic and vanish somewhere, unless the periodic function has a constant component, as in the case of the

chemical potential. Only in the latter case, when the new free-electron FS does not intersect the old FS anywhere, do we have a big window for the inhomogeneous state.

A problem worth studying is one where both a second neighbor term and chemical potential destroy the nested Fermi surface, with the latter as the control parameter. 


\section{THE RENORMALIZATION PROGRAM}

As mentioned earlier, the simple minded and intuitively appealing procedure of eliminating thin slices on either side of the FS works in the case of problems where the FS has special symmetries. The reason, as explained in detail in reference [1], is as follows. When we compute a one loop graph for the $\beta$-function, the two lines in the loop are either particle-hole (in the CDW case) or particle-particle (in the BCS case). To get a nonzero contribution to the flow, it is necessary that both lie in the thin shells being eliminated and also obey momentum conservation. In the BCS case if the overall momentum is zero, these lines have equal and opposite momenta. Given time-reversal symmetry, they have equal energies, so that if one lies in one or the other shell (above or below the FS) that is being eliminated, so does the other, no matter what the direction of the line momentum. In the CDW case, the momenta of the particle and hole lines differ by $Q=(\pi, \pi)$. Since this reverses the energy for the nested problem, again if one lies (anywhere) in the shell below or above the FS that is being eliminated, the other lies in the shell of opposite energy, also being eliminated.

In both cases, no matter how many shells we eliminate, the couplings keep flowing, with each factor of $s$ reduction in cut-off giving a contribution proportional to $\ln s$. The flow is off to the condensed state.

All this changes if we break the symmetry. Consider the CDW example. Now in order for one of the momenta to lie in a shell of thickness $d \Lambda$ and scatter into another one also being eliminated, its momentum must have a very specific direction that lies within a narrow range of size equal to the shell thickness divided by bandwidth. Thus in the shell elimination game, there will be no flow. This is not an insurmountable problem. In reference [1] another scheme called the Field Theory Scheme (FTS) is invoked. Here one computes some physical quantity in the cut-off theory and sets the derivative with respect to the cut-off to zero to

obtain the $\beta$-function. We get the same flow as before for $\Lambda>>$. The flow does change character as $\Lambda$ approaches $I$, as it should, and indicates a transition. But we are not very happy with this method since the FTS is not generally to be used when any of the other 
energies in the problem comes close to $\Lambda$. This is because in the FTS one tries to get away with just the quartic interaction and this requires that the cut-off dominate all other energies. In other words the smallness of ratios like $p / \Lambda$ or $q / \Lambda$ is what allows one to neglect higher operators. In the full fledged Wilson-Kadanoff scheme there is, of course, no such restriction, provided one works with operators of arbitrary complexity, a prospect we do not want to entertain. We are currently engaged in solving this problem.

Another approach is to follow Hertz 13]. Here one couples the fermions to a bosonic variable $\Delta$ as in a Hubbard-Stratanovich transformation, integrates the fermions out and works with the effective action for $\Delta$. As mentioned in Reference [1], this is generally going to cause trouble since integrating out gapless degrees of freedom (anathema to the standard RG) can and typically will, lead to a singular action for the remaining fields. If one did this for the usual BCS problem, one finds that the action cannot be expanded in powers of $\Delta$. Even with the $I$ term present, the action is non polynomial in $\Delta$ since the chosen momentum at the transition to the inhomogeneous phase is a singular point of the susceptibility. (In three dimensions this is not so because of phase space. In one dimension the singularity is even more pronounced.) Even if the action is polynomial, it may not have an analytic expansion in $\omega$ or $q$. Hertz has argued that in some cases we can still find the right scaling when this happens.

Returning to the general problem, one option is to keep both the fermions and bosons together and not eliminate only non-singular modes. This is what one does in problems where gauge fields couple to fermions. In such cases the following question arises. For bosons the energy is measured from the origin in momentum space while for fermions it is measured from the FS. How are high and low energy modes to be defined? A boson that imparts a momentum parallel to the fermions momentum can take it out of the cut-off (and is a high energy boson) while the same boson, if it attaches itself to a fermion with a perpendicular momentum, will move it along the FS and be a low energy boson. A common solution is to pick a point on the fermion's FS and treat bosonic momenta in radial and angular directions distinctly. We find this approach to a rotationally invariant problem 
unsatisfactory, even though for reasons we do not fully understand, it might work. On the other hand a recent paper by Altshuler, Ioffe and Millis [14 describes a class of problems where such a choice of coordinates is warranted. These authors consider a FS which is not rotationally invariant and only two small segments of which (parallel under translation by

a vector $\vec{Q}$ ) are important. In this case there is a preferred fermion direction and using the fermion dispersion relation near these points to decide which bosons are high energy and which are low energy seems legitimate. This is one very promising possibility we intend to fully digest when we resume our RG program. However, for isotropic problems involving bosons and fermions, we believe there is still need for improvement. In the meantime we can take the attitude that since no problem is really isotropic, one may begin by attacking the gauge problems by starting with, say an elliptical FS and trying the scheme of Altshuler et al.

\section{CONCLUSION}

We began with systems that have quantum phase transitions at zero coupling, which exist due to special symmetries of the FS. Two examples given in Reference [1] and discussed here were the planar superconductor (with a time-reversal invariant spectrum) and planar spinless fermions at half-filling (with nesting symmetry of the FS). The goal was to analyze systems with transitions at nonzero, but small, coupling so that a perturbative RG would be possible. To this end we had to come up with systems with such transitions, study them in mean-field to map out the phase structure, and then apply the RG to the transitions. The first step was accomplished by destroying the symmetries of the FS by adding a term $I$ to the hamiltonian. In the superconductor this was a parallel magnetic field and in the spinless fermion case it was a chemical potential, which we saw could be accomplished once again by a parallel magnetic field. The, second step, which led to the results emphasized here, is the

mean-field analysis which showed us the following : 
- The two systems have a very similar three phase structure and phase transitions between them. They have isomorphic formulas for energy and susceptibilities. They naturally have similar windows for the inhomogeneous state. Note that we do not expect the systems to behave identically when fluctuations are considered, since they break different kinds of symmetries.

- The phase at large $I$ (or small coupling) is a Fermi liquid, which is polarized in the superconducting case. The next phase as $I$ is reduced is has a condensate that has a nonzero momentum with respect to the canonical value, this being zero for the superconductor and $(\pi, \pi)$ for the CDW. For this reason we call it the inhomogeneous phase. It has gapless excitations along with the nonzero order parameter. We have computed some of its properties for the superconductor in Section III. The last phase (at small $I$ ) is a state with condensate at the canonical momentum, which is the BCS state for the superconductor and the $Q=(\pi, \pi)$ state for the CDW.

- The transition from the Fermi liquid to the inhomogeneous state is second order in mean-field but with nonclassical exponents: $\beta=2$ for the superconductor. The nonclassical exponents arise because the chosen momentum for the condensate is a singular point of the Fermi liquid susceptibility. The transition from the intermediate state to the homogeneous state is first order.

- The large window for the inhomogeneous state is due to two-dimensionality. Indeed anisotropic systems have larger windows since they are more one-dimensional. Recall that for the CDW case

$$
\frac{\Delta_{0}}{\sqrt{2}}<I<r \Delta_{0}
$$

where $\Delta_{0}$ is the order parameter before any field is applied and $r$ is the anisotropy. For the isotropic superconductor, set $r=1$ above.

We expect a similar enlargement of the window for anisotropic superconductors. 
- In terms of experimental realizations, we require clean, truly two-dimensional, low $Z$ systems.

In the superconducting case we need a $B$ (in Tesla ) of order $T_{c}$ (in Kelvin) assuming a free electron $g$ factor.

In the CDW case, in an idealized world where spinless fermions exist, once again Zeeman energies of the order

of the gap will be needed.

In practice, to employ the trick discussed here, stronger fields may will be needed to separate real electrons into two sufficiently polarized species. Here a large $g$ factor will help.

Our analysis has many limitations. First it is mean-field. The order of the transitions may change. (The analysis of Altshuler et al suggests both transitions may be first order.) However, given the large windows, the intermediate phases should not disappear due to fluctuations.

Our analysis ignores impurities and spin-orbit scattering. To see the phase discussed here will require clean, low $Z$ two-dimensional systems and strong magnetic fields. For the CDW case, where we try to move the FS using the field, a large $g$ factor will help. It appears that we are at the threshold of having all these.

Our analysis is at $T=0$. This restriction was not due to any technical reasons and can be readily overcome.

While our RG program has naturally led us to study this family of systems, the systems themselves are not new. As we mentioned, Bulaevskii has presaged many of our results for the superconductor. There is no doubt a similarly huge body of literature on the CDW problem, both theoretical and experimental. Rather than focus on individual systems, we have taken a slice through them and emphasized the common thread that runs through many of them. The next step is to go through individual cases that have been studied 
experimentally and see if they could belong to this family, i..e, see if they were clean enough, two-dimensional enough, satisfy the various assumptions made in the derivation etc. For example do our consideration possibly apply to granular superconductors? [15] [16] Do they apply to CDW transitions induced by application of pressure? [17] We expect a careful analysis of the huge literature (of which we have touched on a small sample just to make our point) will be quite involved and welcome the readers' input. In the meantime we emphasize that the

experimental detection and study of the

intermediate inhomogeneous phase in any one system will not only be fascinating in its own right, but also in terms of the family structure emphasized here.

\section{Acknowledgements}

We thank V. Ambegaokar, M.Beasely, J.Brooks, A. Goldman, B.I.Halperin, P.Hohenberg, K.Maki, B.Maple, A.Millis, D.R.Nelson, N.Read, H.Schulz, W.Skocpol, S.Sondhi and Y.Liu for helpful discussions on this subject, and are specially indebted to Daniel Prober and Subir Sachdev, for their generous time and assistance. This work was supported by NSF Grants DMR 9311949 and DMR 9120525. 


\section{REFERENCES}

[1] R.Shankar, Rev. Mod. Phys., 66, 239, (1994).

[2] B.S.Chandrasekhar, Appl. Phys. Lett., 1, 7, (1962). A.M.Clogston, Phys. Rev.Lett., 3, 266, (1962).

[3] A.I.Larkin and Yu. N. Ovchinnikov, Soviet Physics JETP, 20, 762, (1965).

P.Fulde and R.A.Ferrel, Phys. Rev. 135, A550, (1964).

[4] For an analysis of this phase in heavy fermion superconductors, see G.Yin and K.Maki, Phys.Rev. B48, 650, (1993).

[5] L.N.Bulaevskii, JETP, 37, Vol. 6, 1133, (1973), ibid 38, Vol. 3, 634, (1974).

[6] S.A. Brazovskii, Sov. Phys. JETP, 41, 85, (1975).

[7] P.C. Hohenberg and J.B.Swift, Metastability in Fluctuation Driven First-order Transitions: Nucleation of lamellar Phases, 1995 Preprint (ATT Bell Labs).

[8] D.E.Prober, R.E.Schwall and M.R.Beasely, Phys. Rev. B21, 2717, (1980).

[9] R.A.Klemm, A.Luther, and M.R.Beasley, Phys.Rev. B 12, 877, (1975).

[10] B.I. Halperin, Physics of Low Dimensional Systems, edited by Y.Nagaoka and S.Hikami, (Publications Office, Prog. in Theoretical Physics, 1979).

D.R.Nelson, Phase transitions and Critical Phenomena, edited by C. Domb and J.L.Lebowitz, (Academic press, New york, 1983, Vol 7).

[11] S.Chakravarty, B.I. Halperin and D.R.Nelson, Phys. Rev. B 39 , 2344 , (1989).

[12] A.V.Chubukov, S.Sachdev and J.Ye, Phys. Rev. B49 , 11909, (1994).

S.Sachdev and T.Senthil and R.Shankar, Phys. Rev. B 50 , 258, (1994).

[13] J.A. Hertz, Phys. Rev. B14, 1165, (1976).

[14] The $T=02 k_{F}$ density wave phase transition in a two dimensional Fermi liquid is first 
order., by B. Altshuler, L.Ioffe, and A.Millis, 1995 preprint.

[15] Y.Liu and A.M.Goldman, Mod.Phys. Lett. B 8, Vol. 8, 277, (1994).

[16] W. Wu and P.W.Adams, Phys. Rev. Lett.,73, 1412, (1994).

[17] M. Regueiro, J. Mignot, and D.Castello, Europhys. Let.18, 53, (1992). 


\section{Figure Captions}

Figure 1. Linear phase diagram for (a) the superconductor and (b) CDW systems as $I$ is raised. In both cases, $I_{0}$ is where the Fermi liquid susceptibility for uniform condensate diverges. It does not correspond to a phase transition. In the CDW case $I_{q}$ is when the Fermi liquid susceptibility diverges for momentum is the $x$ direction. This too is not a real transition since the system has already condensed at $I_{p}$ to a state with momentum in the $y$ direction. All momenta are measured from $(\pi, \pi))$ in the CDW case.

Figure 2. The dark lines show the anisotropic, two-branched, nested Fermi surface at halffilling. The vector $\vec{Q}$ ) connects any point on it to any other point. When the chemical potential is turned on, the surface shrinks to the thin line. There is no global nesting vector. Figure 3. The energy of the the inhomogeneous and uniform BCS states relative to the polarized Fermi liquid (PFL) as a function of the order parameter $\Delta$, just when the BCS energy (labeled $q=0$ ) relative to that of the PFL. vanishes. This is the point $I_{F}=\Delta_{0} / \sqrt{2}$ in Figure 1. Note that that at this point the inhomogeneous state has a lower energy. The optimal momentum is somewhat larger than $I$. Given the energy scales, it clear that under a very slight reduction of $I$, the BCS state will dip below the inhomogeneous state.

Thus we may take $I_{F}=\Delta_{0} / \sqrt{2}$ as the transition point between the uniform state and the inhomogeneous state.

Figure 4. Phase structure as a function of number density $n$ as well as chemical potential I. As $I$ is raised, the system evolves along the $x$ axis from the $(\pi, \pi)$ CDW state to the inhomogeneous state to the PFL. As a function of $n$, slightest doping from half-filling leads to phase coexistence till $n$ comes down to $n_{q}$. Thereafter the behavior is quite smooth. 